\title{
Aplicação de algoritmo evolutivo para reconfiguração de rede de distribuição, visando maior ramificação do sistema
}

\author{
Iulle de M. G. Neves*. Mauro A. G. Clark** \\ Aldir S. Sousa*** \\ * Centro de Tecnologia - Universidade Federal do Piauí (UFPI) \\ 64049-550 - Teresina - PI - Brasil \\ E-mails: iulle@live.com, mauroclark19@hotmail.com \\ ** Centro de Tecnologia e Urbanismo - Universidade Estadual do Piauí (UESPI) \\ 64003-750 - Teresina - PI - Brasil \\ E-mails: aldirsousa@gmail.com
}

\begin{abstract}
This article presents a new approach to the distribution networks reconfiguration problem aiming to obtain a greater branched configuration in order to set the system into a more reliable topology. We have performed analysis of the main changes resulting from this methodology: voltage profile, losses and number of consumers without electricity in case of power failure. The results of simulations, applied to test systems of $16,33,84$ and 136 bars, have shown that by minimizing the maximum level of the network, considering it as a tree, it is possible to achieve greater reliability in the system.

Resumo: Este artigo apresenta uma nova abordagem para o problema de reconfiguração de redes de distribuição visando obter uma configuração com maior ramificação, para conseguir sistemas com topologias mais confiáveis. Realizamos a análise das principais mudanças decorrentes dessa metodologia: perfil de tensão, perdas e número de consumidores desenergizados em caso de falta de energia. Os resultados das simulações, aplicados aos sistemas de teste de 16, 33, 84 e 136 barras, mostraram que, ao minimizar o nível máximo da rede, considerando-a como uma árvore, é possível alcançar maior confiabilidade no sistema.
\end{abstract}

Keywords: Distribution Network, Genetic Algorithm, Ramification, Evolutionary Systems, Losses.

Palavras-chaves: Algoritmo Genético, Perdas, Rede de Distribuição, Ramificação, Sistemas Evolutivos

\section{INTRODUÇÃO}

Os sistemas aéreos de distribuição de energia elétrica que possuem configuração radial têm baixa confiabilidade quanto à continuidade do fornecimento de energia. Isso é devido ao fato de existir apenas um caminho entre a subestação e cada barra (Schmidt, 2005).

Falta de energia traz transtornos aos consumidores e prejuízos financeiros às concessionárias, devido às multas relacionadas aos índices Duração Equiva-lente de Interrupção por Unidade Consumidora (DEC) e Frequência Equivalente de Interrupção por Unidade Consumidora (FEC) (Aneel, 2016). Contingências ocorrem esporadicamente em sistemas de energia e não podem ser evitadas, pois em sua maioria são causadas por intempéries climáticas ou eletromagnéticas (Garcia, 2005). Uma maneira de amenizar o impacto causado pelo problema é diminuir o número de consumidores desenergizados (Aneel, 2016).

Por esse motivo, é essencial que a rede esteja preparada para eventuais contingências. A maneira proposta neste trabalho para alcançar confiabilidade, melhorias no sistema maior capacidade para enfrentar faltas de energia, inevitáveis a uma rede elétrica, é a ramificação.

O objetivo principal deste projeto é obter através do processo de reconfiguração, uma rede de distribuição mais ramificada, visando diminuir o impacto causado por uma contingência. Além disso, examinar consequências advindas dessa metodologia.

\section{MÉTODOS E PROCEDIMENTOS}

Sistemas nos quais as barras estão muito longe da subestação podem possuir elevadas perdas de potência, muito aquecimento nos condutores, perfil de tensão ruim nas últimas barras, entre outros malefícios (Martins,2018). Tudo isso se deve à distância entre subestação e barra, uma vez que o sistema não possua uma boa quantidade de ramificações.

Além dos problemas relacionados ao funcionamento do sistema, é evidente que redes pouco ramificadas sofrem um impacto mais severo com a ocorrência de faltas em suas linhas, como mostra a Fig. 1. 


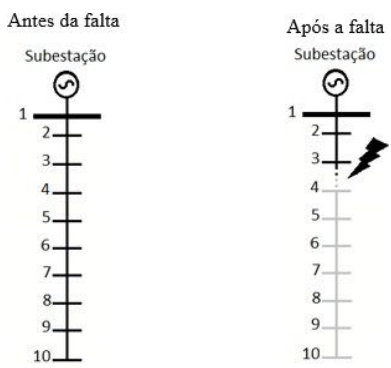

Fig. 1. Sistema com apenas um ramo.

Em sistemas radiais, com a queda de uma linha todo o sistema, além do ponto de contingência, fica desenergizado. Embora a reconfiguração da rede possa reestabelecer o fornecimento de energia, esse processo leva um certo tempo para ser realizado. O cenário ideal, mostrado na Fig. 2, consiste em todos os consumidores ligados diretamente à subestação. Pois na ocorrência de uma falta, apenas uma única barra fique desconectada do sistema.
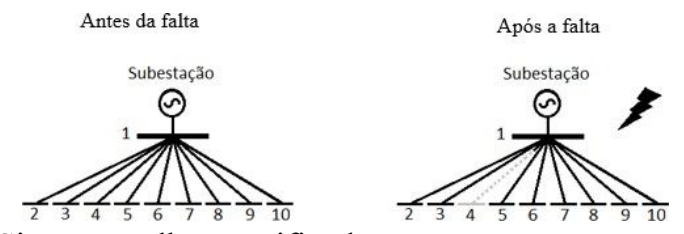

Fig. 2. Sistema melhor ramificado.

Entretanto, é um cenário inviável, visto que: as redes reais não possuem essa estrutura; as redes de distribuição devem ramificar-se pela área atendida, misturando-se com a topologia da cidade (Fonseca, 2016); seriam necessárias muitas subestações nesse formato para atender toda a demanda. Normalmente, sistemas reais não são configurados visando a ramificação, mas como mostrado esse objetivo tem sua importância. Portanto, são necessários métodos eficientes para tornar a rede ramificada, que mantenham a radialidade, dê confiabilidade e robustez ao enfrentar contingências.

A análise feita quanto aos benefícios da ramificação compõe- se da verificação dos níveis de tensão, perda de potência e, principalmente, o número de barras desenergizadas em caso de contingência. Para este estudo, foi realizada a comparação entre os sistemas antes e depois da ramificação.

\subsection{Ramificação}

Para diminuir longos corredores, propõe-se diminuir a profundidade geral da rede. Para melhor entendimento, a seguir, na Fig. 3 é mostrado um exemplo prático da metodologia.

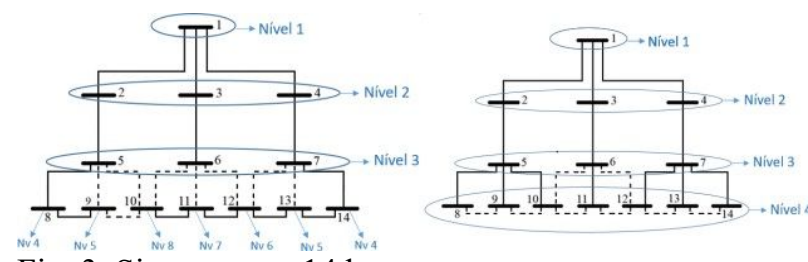

Fig. 3. Sistema com 14 barras.
Conceitualmente, o nível da barra é a profundidade que essa barra se encontra partindo da subestação. Pode-se observar no primeiro cenário que a barra mais distante da subestação é a barra 10 e está no nível 8 , pois ela é a oitava barra a partir da subestação. Já no segundo cenário, a maior profundidade é encontrada nas barras 8 a 14, com nível 4 .

Uma outra análise que pode ser feita é quanto à suposição de uma falta, por exemplo entre as barras 7 e 14 . Antes da ramificação, teremos uma situação em que 5 barras ficam desconectadas, enquanto que para o sistema após a ramificação, somente uma barra fica desconectada. Isso demonstra como a metodologia diminui consideravelmente o impacto causado por contingências.

O processo de ramificação utilizado consiste na minimização do somatório dos níveis das barras da rede, como mostrado em (1).

$$
\operatorname{Min}(P)=\sum_{i=1}^{n} N b_{i}
$$

s.a:

Radialidade

$V i, \min \leq V i \leq V i, \max$

Na qual:

- $\quad P$ é a profundidade do sistema;

- $\quad n$ é o número de barras do sistema;

- $\quad N b$ é o nível da barra;

- $\quad i$ é o índice da barra, variando de $1 \mathrm{a}$.

- $V i, V i, \min$ e $V i$, max correspondem às tensões na barra, mínima e máxima, respectivamente.

\subsection{Algoritmo Genético}

Para encontrar a solução que minimize (1), foi utilizado o Algoritmo Genético (AG). Não foi encontrado na literatura um trabalho visando a ramificação para ser usado como base de comparação da metodologia proposta. Então, como forma de validar o desempenho do algoritmo implementado, antes de usá-lo com o objetivo de ramificar, este foi utilizado para minimização de perda e comparado com pesquisas bem difundidas no meio acadêmico. Os resultados encontrados pelo algoritmo, relacionados com a perda dos sistemas testes utilizados, são mostrados nas Tabelas $1 \mathrm{e} 2$.

Tabela 1.

Comparação de perda de potência sistemas de $\mathbf{1 6}$ barras

\begin{tabular}{|l|c|c|c|}
\hline Sistemas & Configuração & Chaves abertas & Perdas(kW) \\
\hline \multirow{3}{*}{16 barras } & Inicial & $14,15,16$ & 511,44 \\
\cline { 2 - 4 } & $\mathrm{AG}$ & 7,8 e 16 & 466 \\
\cline { 2 - 4 } & $\begin{array}{c}\text { (Cinvalar, } \\
1988)\end{array}$ & 7,8 e 16 & 466 \\
\hline
\end{tabular}


Tabela 2. Comparação de perda de potência sistemas de 33, 84 e 136 barras

\begin{tabular}{|c|c|c|c|}
\hline Sistemas & Configuração & $\begin{array}{l}\text { Chaves } \\
\text { Abertas }\end{array}$ & $\begin{array}{l}\text { Perdas } \\
(\mathrm{kW})\end{array}$ \\
\hline \multirow{3}{*}{33 barras } & Inicial & $\begin{array}{l}33,34,35, \\
36,37\end{array}$ & 202,67 \\
\hline & $\mathrm{AG}$ & $\begin{array}{l}7,9,14,32 \\
\text { e } 37\end{array}$ & 139,55 \\
\hline & $\begin{array}{l}\text { (Mantovani, } \\
\text { 2000) }\end{array}$ & $\begin{array}{l}7,9,14,32 \\
\text { e } 37\end{array}$ & 139,55 \\
\hline \multirow{3}{*}{84 barras } & Inicial & $\begin{array}{l}84,85,86, \\
87,88,89, \\
90,91,92, \\
93,94,95, \\
96\end{array}$ & 531,99 \\
\hline & $\mathrm{AG}$ & $\begin{array}{l}7, \quad 13,34, \\
39,42,55, \\
62,72,83, \\
86,89,90 \\
\text { e } 92\end{array}$ & 469,88 \\
\hline & $\begin{array}{l}\text { (Wang, } \\
\text { 2008) }\end{array}$ & $\begin{array}{l}7,13,34, \\
39,42,55, \\
62,72,83, \\
86,89,90 \\
\text { e } 92\end{array}$ & 469,88 \\
\hline \multirow{3}{*}{$\begin{array}{l}136 \\
\text { barras }\end{array}$} & Inicial & $\begin{array}{ll}136, & 137 \\
138, & 139, \\
140, & 141, \\
142, & 143, \\
144, & 145, \\
146, & 147, \\
148, & 149 \\
150, & 151, \\
152, & 153, \\
154, & 155, \\
156 & \end{array}$ & 320,39 \\
\hline & $\mathrm{AG}$ & 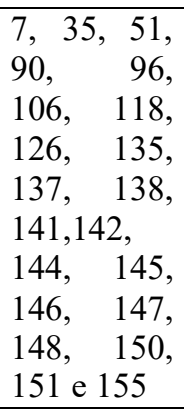 & 280,16 \\
\hline & $\begin{array}{l}\text { (Carreño, } \\
\text { 2008) }\end{array}$ & $\begin{array}{l}\begin{array}{l}7, \\
95,\end{array}, \quad 51, \\
96, \\
106, \quad 118, \\
126, \quad 135, \\
137, \quad 138, \\
141,142, \\
144, \quad 145, \\
146, \quad 147, \\
148, \quad 150, \\
151 \text { e } 155\end{array}$ & 280,16 \\
\hline
\end{tabular}

O AG, inicialmente desenvolvido por Holland (1992), possui funcionamento baseado na Teoria da Evolução das espécies de Charles Darwin, na qual o ser vivo sobrevivente é o que melhor se adapta na natureza. Esta adaptação se dá através de operadores genéticos como recombinação e mutação. As etapas do algoritmo implementado serão explicadas a seguir.

Geração da população: Normalmente, a população é composta por cromossomos com codificação binária. Para aplicação deste artigo, cada gene do indivíduo representa o estado das chaves dos sistemas, portanto, o tamanho do indivíduo foi indicado pela quantidade de ramos de cada sistema teste.

Fitness:Os sistemas utilizados para análise operam com configuração radial, por isso, antes de avaliar os cromossomos, foi realizada a factibilização, checando as restrições de radialidade e conexidade. Foi realizada uma estratégia de factibilização, para evitar o descarte de bons indivíduos, possibilitando melhora na convergência do algoritmo.

O cálculo do fitness aplicado ao problema em questão, considera o indivíduo mais adaptado como sendo o que proporciona o menor somatório de níveis da rede, como já mostrado em (1).

Cruzamento e mutação: Depois da avaliação, indivíduos são selecionados para o cruzamento (troca de material genético) e mutação (alteração genética) (Nascimento, 2015). Os novos indivíduos gerados comporão a nova população, reiniciando assim o ciclo. Abaixo, na Tabela 3, os parâmetros usados no $\mathrm{AG}$ são exibidos, dependendo do sistema.

Tabela 3. Parâmetros do AG

\begin{tabular}{|c|c|c|c|c|}
\hline AG & $\begin{array}{c}16 \\
\text { barras }\end{array}$ & $\begin{array}{c}33 \\
\text { Barras }\end{array}$ & $\begin{array}{c}84 \\
\text { barras }\end{array}$ & $\begin{array}{c}136 \\
\text { barras }\end{array}$ \\
\hline População & 50 & 100 & 200 & 200 \\
\hline Gerações & 50 & 100 & 200 & 300 \\
\hline $\begin{array}{c}\text { Taxa de } \\
\text { cruzamento }\end{array}$ & $100 \%$ & $100 \%$ & $100 \%$ & $100 \%$ \\
\hline $\begin{array}{c}\text { Taxa de } \\
\text { Mutação }\end{array}$ & 0 & 0 & 0 & 0 \\
\hline
\end{tabular}

\section{RESULTADOS}

O processo de reconfiguração de sistemas de distribuição formulado foi implementado utilizando o software Matlab 2015. As simulações foram executadas utilizando um computador com processador Intel Core ${ }^{\mathrm{TM}}$ i7 de $2.7 \mathrm{GHz}$ e $8 \mathrm{~GB}$ de memória RAM; sistema operacional Windows 10. Para atestar e examinar o processo proposto, foram realizados testes em 4 sistemas: 16, 33, 84 e 136 barras, todos operando com potência base de 100 MVA. Para obtenção do perfil de tensão e valores de perda de potência, foi utilizada a biblioteca MATPOWER (Zimmerman, 2011).

Para análise, foi levado em consideração todas as possíveis faltas, número total de consumidores desenergizados, perfil de tensão e perdas ativas. Além disso, foi escolhido o ramo com maior número de barras conectadas da configuração padrão de cada sistema, para supor a falta. A seguir, em (2), está a formulação matemática do cálculo do número total de consumidores desenergizados. 


$$
\text { Desenergizados }=\sum_{i=1}^{m} n_{i}
$$

Na qual:

- m é o número de linhas do sistema;

- $\mathrm{n}$ é o número de barras desenergizadas na falta da linha i;

- $i$ é o índice da linha, variando de $1 \mathrm{a}$ m.

\section{Sistema de 16 barras}

O primeiro sistema teste possui originalmente 16 barras (Cinvalar, 1988), 3 subestações, 13 chaves fechadas e 3 abertas, opera com tensão de $23 \mathrm{kV}$. Para a aplicação proposta, este sistema, como mostrado na Fig. 5, foi utilizado com 14 barras, já que as barras das subestações podem ser modeladas como uma só. Na Tabela 4, são mostradas a configuração padrão, a solução encontrada, as respectivas perdas e número total de consumidores desconectados supondo todas as possíveis faltas.

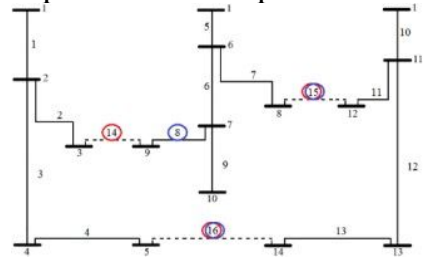

Fig. 5. Sistema de 16 barras.

\section{Tabela 4. Resultados encontrados para o sistema de} 16 barras

\begin{tabular}{|l|l|l|}
\hline Configuração & Padrão & Proposta \\
\hline Chaves Abertas & 14,15 e 16 & $8,15,16$ \\
\hline Fitness & 41 & 41 \\
\hline Perdas (kW) & 511,44 & 493,15 \\
\hline Desenergizados & 27 & 27 \\
\hline
\end{tabular}

Com a configuração padrão já era possível encontrar a máxima ramificação, porém o algoritmo encontrou outra solução que proporciona menor perda ao sistema, redução de $3,58 \%$.

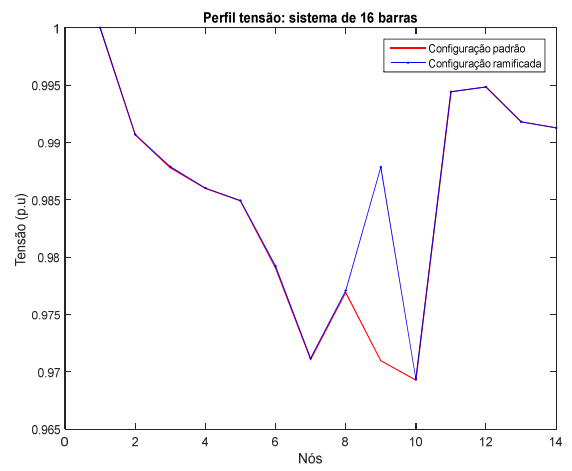

Fig. 6. Perfil de tensão da configuração padrão (vermelho) e da solução (azul) do sistema de 16 barras.
Percebe-se que, os níveis de tensão foram semelhantes para ambas as configurações, porém a configuração proposta obteve melhor tensão na barra 9 .

\section{Sistema de 33 barras}

O segundo sistema possui 33 barras (Baran, 1989), uma subestação, 32 chaves fechadas, cinco abertas e pode ser observado na Fig. 7. O dado de operação relacionado a tensão é $12.66 \mathrm{kV}$. Na Tabela 5, são mostradas a configuração padrão, a solução encontrada, as respectivas perdas e número total de consumidores desconectados supondo todas as possíveis faltas.

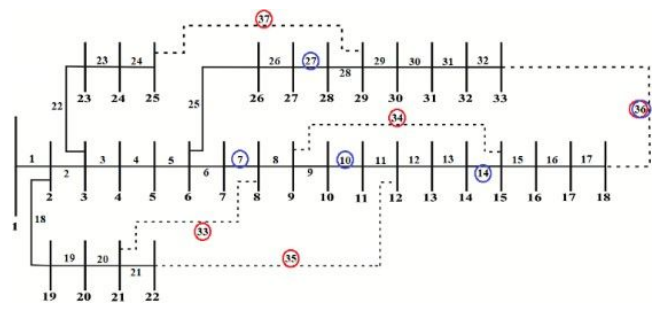

Fig. 7. Sistema de 33 barras.

Tabela 5. Resultados encontrados para o sistema de 33 barras

\begin{tabular}{|l|l|l|}
\hline Configuração & Padrão & Proposta \\
\hline $\begin{array}{c}\text { Chaves } \\
\text { Abertas }\end{array}$ & $33,34,35,36,37$ & $7,10,14,27,36$ \\
\hline Fitness & 288 & 220 \\
\hline Perdas (kW) & 202,67 & 145,96 \\
\hline Desenergizados & 255 & 187 \\
\hline
\end{tabular}

O AG foi capaz de encontrar topologia que reduz em até $28 \%$ o valor das perdas ativas e em $26,67 \%$ o número total de consumidores desconectados, conforme (2).

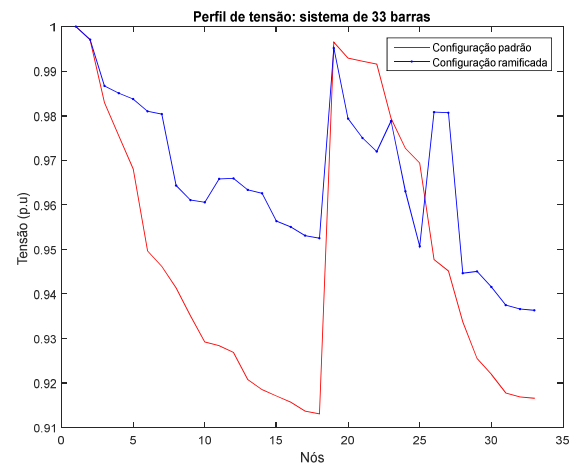

Fig. 8. Perfil de tensão da configuração padrão (vermelho) e da solução (azul) do sistema de 33 barras.

O valor de tensão mínimo da topologia padrão foi 0,9131 , enquanto na solução foi 0,9363 . Em $84,85 \%$ das barras, o nível de tensão da solução foi maior.

\section{Sistema de 84 barras}

No terceiro sistema teste (Chiou, 2005) há 84 barras, 96 circuitos de interconexão, sendo 13 abertos e 83 fechados e pode ser observado na Fig. 9. Este sistema tem como 
tensão base $11,4 \mathrm{kV}$. Na Tabela 6 , são mostradas a configuração padrão, a solução encontrada, as respectivas perdas e número total de consumidores desconectados supondo todas as possíveis faltas.

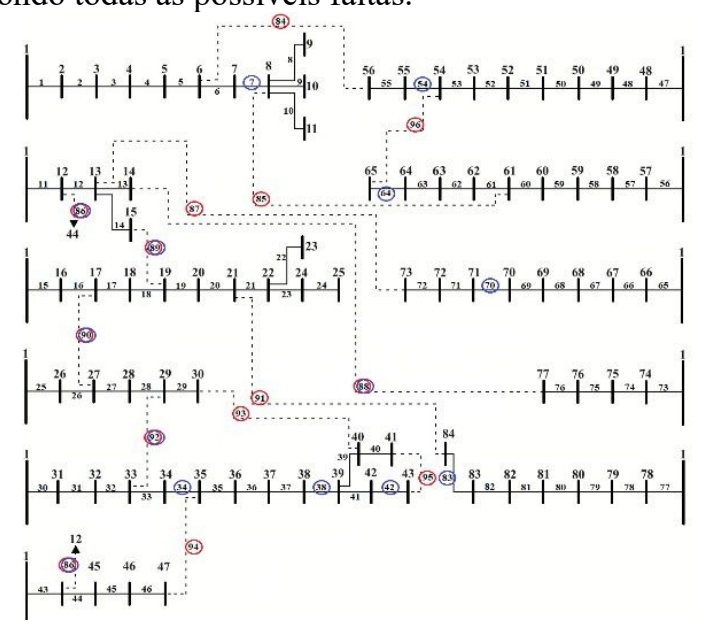

Fig. 9. Sistema de 84 barras.

Tabela 6. Resultados encontrados para o sistema de 84 barras

\begin{tabular}{|c|c|c|}
\hline Configuração & Padrão & Proposta \\
\hline Chaves Abertas & $\begin{array}{l}84,85,86,87, \\
88,89,90,91, \\
92,93,94,95, \\
96\end{array}$ & $\begin{array}{l}7,34,38,42,54,64, \\
70,83,86,88,89 \\
90,92\end{array}$ \\
\hline Fitness & 474 & 441 \\
\hline Perdas $(\mathrm{kW})$ & 531,99 & 546,6 \\
\hline Desenergizados & 390 & 357 \\
\hline
\end{tabular}

A resposta encontrada pelo $\mathrm{AG}$ aumentou em apenas $2,74 \%$ as perdas, e diminuiu em $8,46 \%$ o número total de consumidores desconectados supondo todas as possíveis faltas.

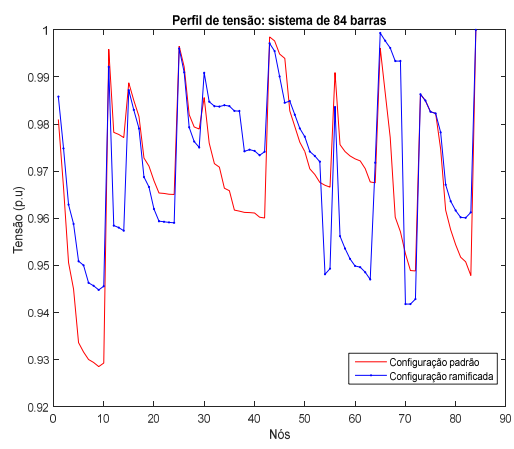

Fig. 10. Perfil de tensão da configuração padrão (vermelho) e da solução (azul) do sistema de 84 barras.

Para este sistema, 51,2\% das barras da solução tiveram nível de tensão melhor que as da configuração padrão, sendo o valor mínimo 0,9418 na primeira e 0,9285 na última.

\section{Sistema de 136 barras}

Este sistema (Mantovani, 2000) é real, localizado em uma cidade do Brasil. Possui as seguintes características: 136 barras, 156 ramos, opera com tensão base $13.8 \mathrm{kV}$ e possui
21 chaves abertas e 135 fechadas e pode ser observado na Fig. 11. Na Tabela 7, são mostradas a configuração padrão, a solução encontrada, as respectivas perdas e número total de consumidores desconectados supondo todas as possíveis faltas.

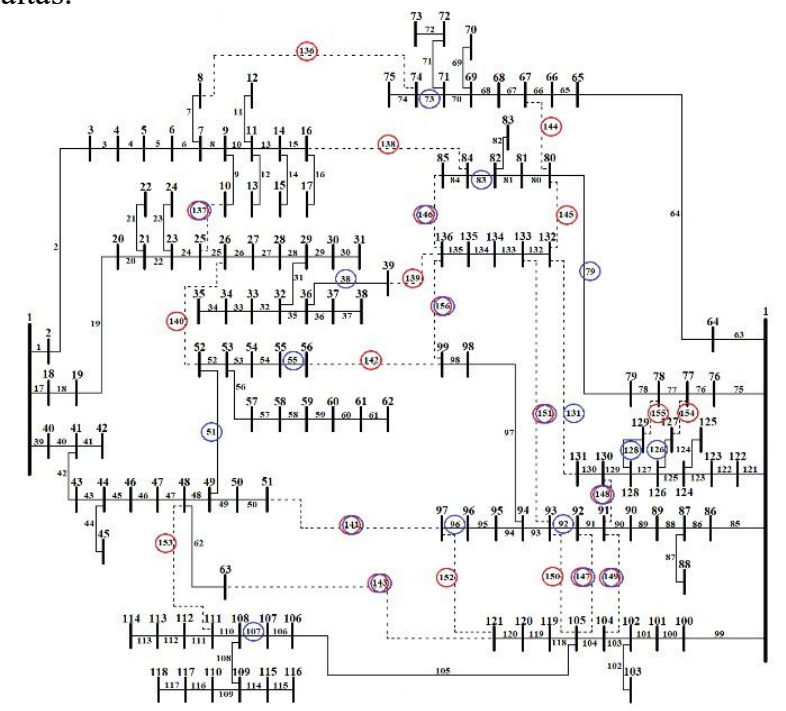

Fig. 11. Sistema de 136 barras.

\section{Tabela 7. Resultados encontrados para o sistema de} 136 barras

\begin{tabular}{|l|l|l|}
\hline Configuração & Padrão & Proposta \\
\hline & $136,137,138$, & $38,51,55,73$, \\
& $139,140,141$, & $79,83,92,96$, \\
& $142,143,144$, & $107,126,128$, \\
& $145,146,147$, & $131,137,141$, \\
& $148,149,150$, & $143,146,147$, \\
& $151,152,153$, & $148,149,151$, \\
& $154,155,156$ & 156 \\
\hline Fitness & 1216 & 1180 \\
\hline Perdas (kW) & 320,39 & 345 \\
\hline Desenergizados & 1080 & 1044 \\
\hline
\end{tabular}

A resposta encontrada pelo AG aumentou em 7,72\% as perdas, e diminuiu em $3,33 \%$ o número total de consumidores desconectados supondo todas as possíveis faltas.

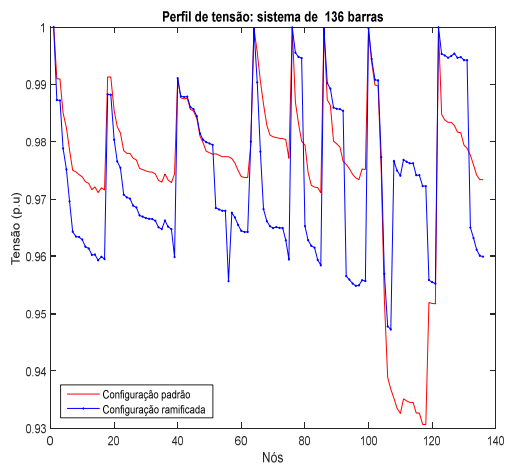

Fig. 12. Perfil de tensão da configuração padrão (vermelho) e da solução (azul) do sistema de 136 barras. 
Em 41,18\% das barras da configuração proposta os valores de tensão foram melhores. Os níveis de tensão mínimo e máximo são 0,9472 e 1 para a solução e 0,9306 e 1 para a configuração anterior a reconfiguração.

\section{ANÁLISE DE RESULTADOS}

Percebe-se que para os sistemas de menor porte (16 e 33 barras) o algoritmo de ramificação encontrou uma perda de potência menor do que a configuração padrão. Isso ocorreu em virtude de o processo de ramificação ter dividido longos trechos, causadores de grandes perdas, em trechos menores.

Por outro lado, para os sistemas maiores (84 e 136 barras), as perdas encontradas foram maiores do que na configuração original. Isso se deve ao fato de que ao aplicar o AG com o objetivo de diminuir a profundidade do sistema, o algoritmo forçou a utilização de linhas com maior impedância, provocando uma perda maior.

Para os sistemas de 16, 33 e 84 barras, o perfil de tensão melhorou em boa parte das barras, já para o sistema de 136 barras, a melhora ocorreu, mas em poucas barras da rede.

É pertinente fazer uma análise em relação ao número de consumidores desconectados em caso de falta, foco principal desse trabalho.

No sistema de 16 barras, ocasionando o desligamento entre as barras 1 e 6, 5 (cinco) barras ficam desenergizadas no sistema inicial e somente 4 (quatro), na solução do problema. Porém, ocorre o aumento de consumidores desconectados, caso fosse suposto falta em outras linhas do sistema ramificado, ratificando os fitness de valores iguais.

Para o sistema de 33 barras, considerando todas as possíveis faltas, ocorre diminuição de 68 consumidores desconectados quando comparadas às configurações proposta e padrão. Supondo-se especificadamente uma falta entre as barras 3 e 4, 23 barras ficam desconectadas na topologia padrão e somente 6 na topologia proposta, diminuindo em 73,91\% o impacto.

No sistema de 84 barras, há diminuição de 29 consumidores desconectados partindo da configuração padrão para a proposta, considerando todas as possíveis faltas. Considerando-se uma contingência entre as barras 31 e 32, 13 barras ficam desconectadas na configuração original e somente 3 (três) barras ficam desenergizadas no sistema ramificado, ou seja, 76,92\% de diminuição de prejuízo.

No sistema de 136 barras, desconectando a linha entre as barras 43 e 45 , ficam 18 barras desconectadas na topologia inicial, já na solução encontrada do problema, 7 barras ficam desconectadas, redução de $61,11 \%$. Supondo todas as faltas, o número de consumidores desconectados diminui em 36, comparando as topologias padrão e proposta.

\section{CONCLUSÕES}

Neste artigo foi proposta uma metodologia aplicada a reconfiguração de rede de distribuição, desejando obter sistemas com maior ramificação possível.

O processo usado possibilitou melhor redistribuição de ramos, realocando barras pertencentes a grandes corredores, em menores trechos. Com a ramificação, foi visualmente detectável a eficiência da proposta, ao observar-se o número de cargas desconectadas após uma contingência.

É importante ressaltar que o algoritmo implementado objetivou unicamente a minimização da profundidade da árvore. Propõe-se para trabalhos futuros a utilização de um algoritmo multiobjectivo para a minimização simultânea da profundidade e das perdas do sistema devido as vantagens econômicas.

\section{AGRADECIMENTOS}

Os autores gostariam de agradecer a Coordenação de Aperfeiçoamento de Pessoal de Nível Superior (CAPES)/ Fundação de Amparo à Pesquisa do Estado do Piauí (FAPEPI) pelo apoio ao projeto que originou esta pesquisa.

\section{REFERÊNCIAS}

ANEEL. Agência Nacional de Energia Elétrica. Audiência Pública $n^{\circ}$. 033/2009 - Revisão do Procedimentos de Distribuição (PRODIST). Brasília, DF, 2016g. Disponível em:

$<$ http://www2.aneel.gov.br/arquivos/PDF/DIC\%20FI C \%20DMIC.pdf $>$.

Baran, M. E.;Wu, F. F., (1989) Network reconfiguration in distribution systems for loss reduction and load balancing. IEEE Transactions on Power Delivery, vol. 4, no. 2, p. 1401-1407.

Carreño, E.; Romero, R.; A. P. Feltrin, (2008). An efficient codification to solve distribution network reconfiguration for loss reduction problem. IEEE Transactions on Power Systems, New York, v. 23, n. 4, p. 1542-1551.

Chiou, J. P.; Chang, C. F.; Su, C. T. (2005). Variable scaling hybrid differential evolution for solving network reconfiguration of distribution systems. IEEE Transactions on Power Systems, New York, v. 20, n .2 , p. 668-674.

Cinvalar, S.; J. J. Grainger,; H. Yin, and S. S. H. Lee, (1988). Distribution feeder reconfigurationfor loss reduction, IEEE Trans. Power Delivery, Vol. III, No. 3, pp. 1217- 1223 .

Fonseca, A. G., (2006). Análise de reconfiguração de redes de distribuição baseada em fluxo de potência desacoplado rápido. M.S. thesis, Universidade Federal do Paraná, PR, BR.

Garcia, V. J., (2005). Metaheurísticas multiobjetivo para o problema de restauração do serviço em redes de distribuição de energia elétrica., Ph.D. Thesis, Universidade Estadual de Campinas, Campinas, SP, BR.

Holland, J. H., (1992). "Adaptation in Natural \& Artificial Systems". Press $2^{\text {nd }}$ ed.: MIT Press.

Mantovani, J. R. S.; Casari, F.; Romero, R. A., (2000). Reconfiguração de sistemas de distribuição radiais utilizando o critério da queda de tensão. Revista SBA: Controle \& Automação, vol. 11, no. 3, set., out., nov., dez.

Martins, C. C. C., (2018). Fluxo de carga em redes de distribuição operando em emergência, M.S. thesis, Universidade Federal do Maranhão, MA, BR.

Nascimento, L. B. P.; Amora, M. A. B.; Pinto. V. P.; D. A. Souza, (2015). Busca Harmônica com ajuste 
adaptativo de parâmetro por meio de Algoritmo Genético aplicada na otimização do projeto LQR. In: Congresso Brasileiro de Inteligencia Computacional. Curitiba-PR: ABRICOM.

Schmidt, H. P., (2005). Reconfiguração de redes de distribuição através de programação não-linear inteira mista., Ph.D. Thesis, Universidade de São Paulo, SP, BR.

Wang, C.; Cheng, H. Z., (2008). Optimization of network configuration in large distribution systems using plant growth simulation algorithm. IEEE Transactions on Power Systems, New York, v. 23, n. 1, p. 119-126.

Zimmerman, R. D., Murillo-S'anchez, C.E., Thomas, R. J, (2011): Matpower: Steady-state operations, planning, and analysis tools for power systems research and education.IEEE Transactions on Power Systems 